\title{
Barriers to Internal Rotation from Relative Intensity Measurements as a Function of Temperature on Microwave Absorption Lines
}

\author{
G. RUITENBERG* \\ Fysisch Laboratorium, Rijksuniversiteit Utrecht, The Netherlands
}

\begin{abstract}
A variant is presented of the relative intensity method (RIM) in microwave spectroscopy to determine barriers to internal rotation. In this method torsional energies are determined directly from the slope of the straight line relating the logarithm of the intensity ratio and the reciprocal of the temperature. The advantage of this simple and direct method is that unknown theoretical or experimental weight factors are cancelled out in the determination of torsional energies from the measured intensity ratios. Using a newly developed Stark absorption cell without a mid-electrode, this RIM has been applicated to the molecules $\mathrm{CH}_{3} \mathrm{SiH}_{3}, \mathrm{HCCOCH}_{3}$ and $\mathrm{HCCSCH}_{2} \mathrm{D}$. The results of the height of the barriers to internal rotation in these molecules are in agreement with the values obtained from frequency splitting methods (FSM). Furthermore, the accuracy obtained in the RIM (3-5\%) can compete with that of the FSM $(2-4 \%)$.
\end{abstract}

\section{INTRODUCTION}

In microwave spectroscopy mainly two methods are utilized to determine potential barriers to internal rotation (torsional vibration). The first method is called the relative intensity method (RIM). It depends on the fact that the intensity ratio $\left(I_{0} / I_{v}\right)$ of a given rotational transition in the ground torsional state to that in an excited torsional state $v$ is proportional to the relative populations of the two torsional states. Assuming a Boltzmann population distribution, the energy difference $E_{v}-E_{0}$ between the two torsional states can be calculated from the formula

$$
I_{0} / I_{v}=f \exp \left|\left(E_{v}-E_{0}\right) / k T\right|,
$$

where $f$ is a proportionality constant.

The energy differences $E_{v}-E_{0}$ for various $v$ values can be brought into relation with the shape and the height of the potential barrier. The virtue of the RIM lies in its theoretical simplicity and its applicability to all types of molecules. However, relative intensity measurements may be subject to rather

* Present address: AKZO Research Laboratories, Arnhem, The Netherlands. 
serious and unpredictable errors, mainly due to frequency-dependent reflection within the absorption cell, and nonlinearities in the detection system (1). These crrors result usually in an aceuracy of about $10-20 \%$ in the barrier height.

A careful design of the absorption cell and application of the "intensity law," derived by Harrington (2), provide a method to measure energy differences with an accuracy of about $5 \%$. However, this method depends on the knowledge of the microwave power density distribution in the sample. Unknown variations of this distribution can result in larger errors in molecular quantities calculated from relative intensity data. Therefore, if possible, information about the potential barrier is usually obtained from the frequency splitting of a rotational transition in a torsional state, due to removal of degeneracy between torsional sublevels ( $A$ or $E$ ), provided that rotational spectra in different torsional states are well separated. With this frequency splitting method (FSM), an accuracy of about $1-4 \%$ is obtainable. The splitting depends on the height of the potential barrier as well as on the rotational and torsional states involved in the transitions and can be calculated by an approximate solution of the Schrödinger equation for the molecule, treating the term describing the interaction between overall and internal rotation as a perturbation term in the Hamiltonian. However, application of this FSM is limited to molecules which show a measurable splitting in the microwave spectrum and whose Schrödinger equations can be solved with sufficient accuracy.

Since the RIM, unlike the FSM, can be applied to all types of molecules performing internal rotation, we have tried to explore the potentialities of the RIM, taking advantage of the special technique of accurate intensity measurements - the antimodulation method-developed in our laboratory by Dymanus and Dijkerman (3). This technique eliminates the possible nonlinearity of the detector.

In order to further improve the RIM, special attention was paid to reduction of the errors introduced by the reflections in the absorption cell. A great reduction of these reflections was realized by means of a new type Stark-absorption cell (4) without a mid-electrode, which also makes possible intensity-ratio measurements as a function of lemperalure in the range from 225 to $325 \mathrm{~K}$. The great advantage of intensity-ratio measurements as a function of temperature is that the energy differences between the ground and excited torsional states can be determined directly from the slope of the curve relating the logarithm of the intensity ratio and the reciprocal of the temperature

$$
\frac{d \ln \left(I_{0} / I_{n}\right)}{d(1 / \mathrm{k} T)}=E_{r}-E_{0} .
$$

In this way unknown theoretical or experimental weight factors included in the proportionality constant $f$ are eliminated from the calculation of the energy differences. 
Using the new absorption cell, we have applied this variant of the RIM to three different types of molecules performing internal rotation, namely methyl silane, $\mathrm{CH}_{3} \mathrm{SiH}_{3}$, symmetric group-symmetric group, methoxyethyne, $\mathrm{HCCOCH}_{3}$, asymmetric group-symmetric group, mono-D-methylthioethyne, $\mathrm{HCCSCH}_{2} \mathrm{D}$, asymmetric group-asymmetric group. The results for the barrier heights in these molecules from intensity-ratio measurements as a function of temperature are compared with those from the FSM to test the capability of the RIM and at the same time to check the theoretical assumptions underlying the FSM.

\section{EXPERIMENTAL ASPECTS OF RELATIVE INTENSITY MEASUREMENTS AS A FUNCTION OF TEMPERATURE}

The quantity usually measured in relative intensity measurements is the ratio of the top intensities of two absorption lines. This ratio $R$ for a given rotational transition in the ground and an excited torsional state at a temperature $T$, can be written as

$$
R \equiv I_{0} / I_{v}=\frac{l_{\text {eff }}}{l_{\text {eff } v}} \frac{g_{0}}{g_{v}} \frac{|\langle i|\mu| j\rangle|_{0}^{2}}{|\langle i|\mu| j\rangle|_{v}^{2}} \frac{\nu_{0.0}^{2}}{\nu_{0 . v}^{2}} \frac{\Delta \nu_{v}}{\Delta \nu_{0}} e^{\left(E_{v}-E_{0}\right) / k T} \equiv f e^{\left(E_{v}-E_{0}\right) / k T},
$$

where the subscripts 0 and $v$ refer to the torsional states of the two lines involved in the intensity measurements; $l_{\mathrm{eff}}$ is the effective path length of the absorption cell; $g$ is a statistical weight factor; $\langle i|\mu| j\rangle$ is the dipole moment matrix element for the rotational transition $i \rightarrow j ; \nu_{0}$ is the resonance frequency; $\Delta \nu$ is the half-width of the line at half intensity and $E_{v}-E_{0}$ is the energy difference between the two torsional states involved.

In order to obtain an accurate value for $E_{v}-E_{0}$ from the intensity ratio $R$, the proportionality factor $f$ must be known with sufficient accuracy. However, a number of the quantities comprised in the expression for the top-intensities may be unequal for the two lines, and a correction for these differences may not always easily be made. For instance, the dipole moment as well as the asymmetry parameter of the molecule may be different for the torsional states, so that extra attention has to be paid to the determination of the dipole-moment matrix element. Furthermore, an unique determination of the ratio of the effective path lengths is very difficult, since any reflections present in the absorption cell may cause irregular changes in $l_{\text {eff }}$ as a function of the microwave frequency. These variations of $l_{\text {eff }}$ mostly are the main source of error in the coefficient $f$ and thus in the energy difference $E_{v}-E_{0}$. However, this difficulty is eliminated for the greater part if it can be assumed that the ratio of $l_{\text {eff }}$ does not vary with temperature. Then $f$ is independent of the temperature $T$, because, to a high order of accuracy, the ratios of $g,|\langle i|\mu| j\rangle|^{2}, \nu_{0}$ and $\Delta v$ respectively, are independent of $T$. The energy difference between two torsional states then can be determined directly from the intensity ratio $R$ measured as a function of 
temperature, without knowledge of the quantities comprised in the factor $f$ [see Eq. (2)].

In practice, however, always some reflections are present in the absorption cell. These reflections cause a part of the microwave radiation to be reflected back and forth several times in the cell. When the geometrical length of the cell varies due to a change in the temperature, the effective path length thus becomes a function of temperature. It can be shown (see Section IV) that the ratio of

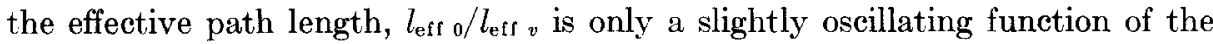
temperature, if sufficient care is taken to minimize the reflections in the absorption cell (5). When intensity-ratio measurements are performed over a temperature range extending over at least one period of this function-in our case about $100 \mathrm{~K}$-, these variations of $l_{\text {eff }} / l_{\text {eff }} v$ do not influence the mean value of the slope of the straight line relating $\ln R$ and $1 / k T$; they only contribute to the random error in the slope, and thus in $E_{r}-E_{0}$.

\section{DESCRIPTION OF THE SPECTROMETER}

With the exclusion of the absorption cell, the spectrometer used to measure relative intensities is a conventional Stark-effect microwave spectrometer. The modulation frequency is $1500 \mathrm{~Hz}$. A long-term microwave frequency stability of about $2 \times 10^{-9}$ is achieved by phase-locking the klystron frequency to harmonics of a uhf (470-1000 MHz) standard frequency generator (Rohde and Schwartz XUC). This stability is sufficient to remain on the top of an absorption line during an intensity measurement.

Determination of the sample pressure is performed with a calibrated mcmbrane manometer.

The relative intensities are measured according to the antimodulation method (3). The antimodulator is a vibrating attenuator. Because of the low modulation frequency, a bolometer is used as the detector.

In order to perform accurate relative-intensity measurements as a function of temperature, it is necessary that the absorption path length of the Stark cell is almost independent of the microwave frequency and temperature. So, it is important to utilize an absorplion cell with a reflection coefficient as low as possible. For that purpose, a new type of Stark cell was developed without a mid-electrode (4). Its principal feature is the insulation of one of the broad walls of the waveguide by teflon tape from the rest of the cell (see Fig. 1). The Stark field is applied to this insulated top plate. Coolant tubes are attached to the top plate and the gutter, which makes it possible to vary the temperature of the cell from 225 to $325 \mathrm{~K}$. The whole cell and cooling tubes are enclosed in a vacuum-tight brass tube, which thermally insulates the Stark cell from the surroundings and provides an electrical shielding for the Stark voltage. Teflon blocks support the cell in the enclosing tube. The cell is vacuum sealed by mica windows and connected to K-band waveguide by tapered transition sections of $19 \mathrm{~cm}$ length. 


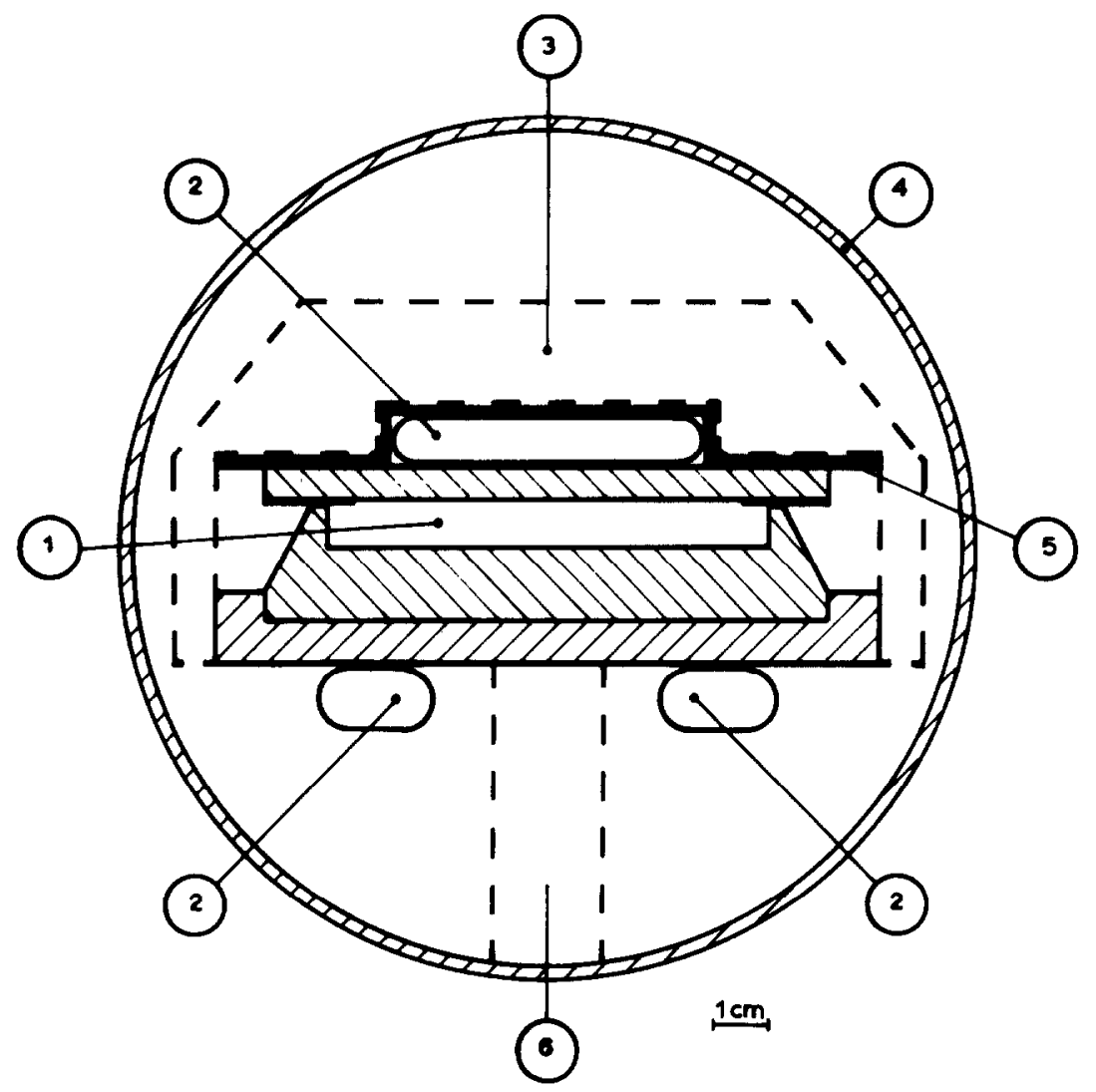

Fia. 1. Cross section of the Stark cell: (1) Absorption cell; (2) coolantconduits; (3) brass clamp; (4) vacuum-tight brass tube; (5) Teflon insulation; (6) supporting block of Teflon.

IV. A TEST OF THE CAPABILITY OF THE SPECTROMETER FOR RELATIVE INTENSITY MEASUREMENTS

\section{Vibrational Energies of Trimethylene Oxide}

The intensity capability of our spectrometer was tested by applying the RIM to the determination of vibrational energy differences that are accurately known from independent measurements. This test was realized by performing relative intensity measurements on rotational transitions of the planar ring molecule trimethylene oxide $\left(\mathrm{C}_{3} \mathrm{H}_{6} \mathrm{O}\right)$ in various excited states of the ring-puckering vibration. From these measurements, energy differences between vibrational states were determined. These vibrational energies have already been determined directly from far-infrared measurements (6), and the accuracy obtained with the latter method is much better than can be realized by our method. So, by comparing the results from the far-infrared measurements with those from rela- 
TABLE I

Rotational Transitions (Frfquencies in MHz) and Vibrational Engrgifs (in $\mathrm{cm}^{-1}$ ) of Trimethylenfe Oxide

\begin{tabular}{|c|c|c|c|c|c|}
\hline \multirow{2}{*}{$\frac{\text { Transition }}{J_{K_{-1} K_{1}} \rightarrow_{J_{K_{-1} K_{1}^{\prime}}^{\prime}}}$} & \multicolumn{3}{|c|}{ Ring-puckering vibrational state } & \multicolumn{2}{|c|}{ Vibrational energy } \\
\hline & $z=0$ & $y=1$ & $i=2$ & $E_{1}-E_{0}$ & $E_{2}-E_{0}$ \\
\hline (1) $5_{32} \rightarrow 5_{51}$ & 18346.37 & 18452.02 & 18490.40 & $52 \pm 5$ & $145 \pm 5$ \\
\hline (2) $7_{53} \rightarrow 7_{52}$ & 24592.65 & 24241.35 & 24070.59 & $55 \pm 4$ & $150 \pm 5$ \\
\hline (3) $1_{11} \rightarrow 2_{12}$ & 31925.96 & 32043.16 & 32085.96 & $53 \pm 6$ & $151 \pm 7$ \\
\hline (4) $1_{10} \rightarrow 2_{11}$ & 41932.65 & 41951.65 & 41945.55 & $50 \pm 7$ & $146 \pm 7$ \\
\hline \multicolumn{4}{|c|}{ Mean value and standard deviation } & $53 \pm 2$ & $147 \pm 3$ \\
\hline \multicolumn{4}{|c|}{ From far-infrared spectrum } & $53.4 \pm 0.2$ & $143.3 \pm 0.2$ \\
\hline
\end{tabular}

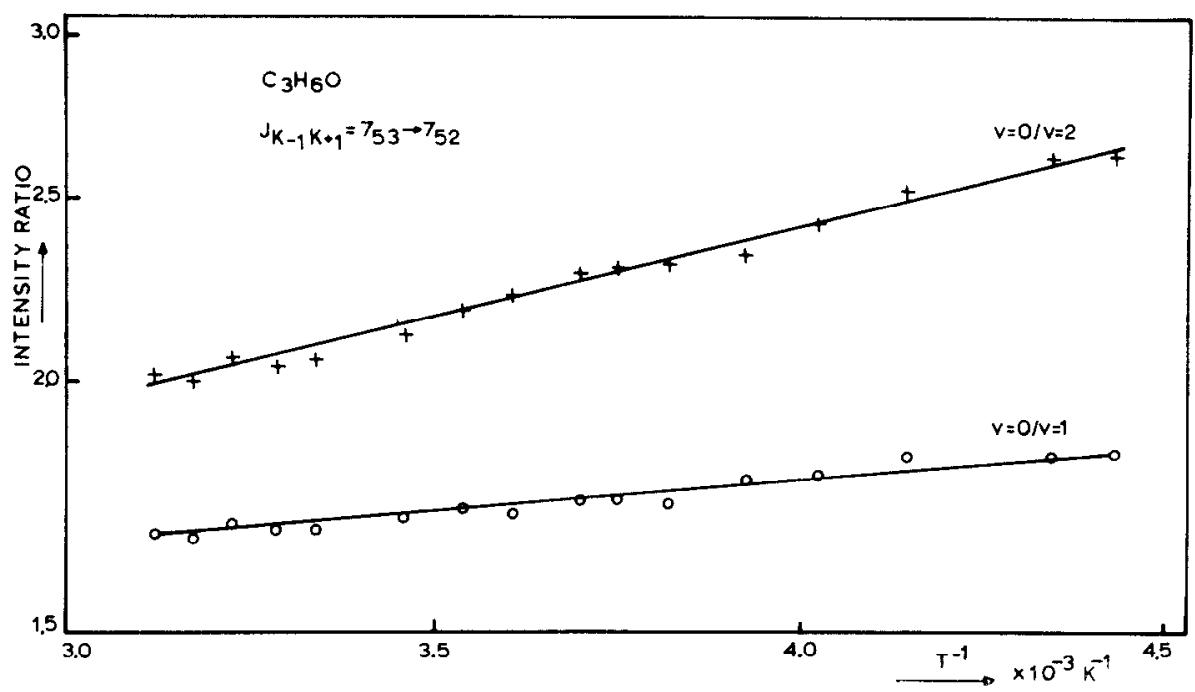

FIG. 2. The intensity ratios $I_{0} / I_{1}$ and $I_{0} / I_{2}$ as a function of $T^{-1}$. The straight lines were obtained by a least-square fit.

tive intensity measurements we could check the precision and reliability of the RIM for the determination of potential barriers.

Relative intensity measurements as a function of temperature were performed on the rotational transitions of $\mathrm{C}_{3} \mathrm{H}_{6} \mathrm{O}$ listed in Table I. The top intensity of each line was measured as a function of temperature in the range from 228 to $323 \mathrm{~K}$. Figure 2 shows, as an example, the results of the measurements on transition (2). It gives the intensity ratios of the satellite lines with respect to the main line as a function of temperature. Using Eq. (2) the vibrational energy differences $E_{v}-E_{0}$ and their standard deviations were calculated for each of the four 
rotational transitions with a least-square fit of the straight line relating $\ln R$ and $1 / k T$.

The results for $F_{v}-E_{0}$ are also collected in Table I. In addition, Table I gives the precise values of $E_{v}-E_{0}$ determined directly from far-infrared measurements $(6)$.

\section{Discussion of Errors}

From the results given in Table I it appears that the vibrational energies of the molecule $\mathrm{C}_{3} \mathrm{H}_{6} \mathrm{O}$ determined with $\mathrm{RIM}$ are in fair agreement with the more accurate values determined directly from the far-infrared spectrum of the molecule. The quoted standard deviations represent the random errors involved in the RIM. A discussion of these random errors and of the influence of possible systematic errors will now be given.

In the antimodulation method it is possible to determine the modulation depth $m$ with an accuracy of $1 \%$, provided $m$ is large with respect to the minimum detectable modulation depth. This results in a relative error of $R$ of about $1.5 \%$.

The systematic error in $R$ caused by the variation of $l_{\text {eff }}$ as a function of both the microwave frequency and the temperature of the absorption cell can be approximated in the following way. Considering a model of a waveguide absorption cell with small equal reflections occurring at both ends, it can be shown that the effective path length can be written as (5)

$l_{\text {eff }}=l\left[1-r^{2} \exp (-2 \gamma 1)\right] /\left[1-2 r \exp (-\gamma l) \cos 2 k l+r^{2} \exp (-2 \gamma l)\right]$,

where

$l=$ radiation path length of the cell

$r=$ microwave power reflection coefficient at the discontinuity regions

$\gamma=$ power attenuation coefficient of the cell

$k=2 \pi / \lambda_{o}$, with $\lambda_{g}$ the radiation wavelength in the cell.

The reflection coefficient $r$ can be determined from the periodic variation of the VSWR of the cell as a function of frequency. Equation (4) then gives an estimate of the variation of $l_{\text {eff }}$ as a function of the radiation path length $l$, and so of the temperature $T$ as $l(T)=l_{0}\left[1+C_{e}\left(T-T_{0}\right)\right]$, where $C_{e}=$ coefficient of linear expansion. In a typical case we found $l=300 \mathrm{~cm}, r=10^{-2}, \gamma=1.2 \times$ $10^{-3} \mathrm{~cm}^{-1}, \nu=24.6 \mathrm{GHz}$ and a period of the oscillating function $l_{\mathrm{eff}}(T)$ of about $100 \mathrm{~K}$.

Measurements of the VSWR of the absorption cell at frequencies near 20 and $30 \mathrm{GHz}$ showed a variation of the VSWR between 1.10-1.25 and 1.20-1.55, respectively. These values result in a maximum variation of $l_{\text {eff }}$ in these regions of about $2 \%$ and $4 \%$, respectively. The expected error of the ratio $l_{\text {eff } 0} / l_{\text {eff }} v$ is then about $1.5 \%$ near $20 \mathrm{GHz}$ and $2.5 \%$ near $30 \mathrm{GHz}$. 
It should be noted that an additional variation of $l_{\text {eff }}$ can occur due to the fact that the reflection coefficient $r$ is a slow function of the frequency. This introduces a constant factor in the intensity ratio $R$ of two absorption lines with a large frequency separation. However, this systematic error is cancelled out in the determination of $E_{v}$ from $R$ as a function of temperature.

It can thus be concluded that the persisting contribution of $l_{\text {eff }}$ to the error of the intensity ratio is due to the oscillating variation of $l_{\mathrm{eff}}$ as a function of temperature. This error increases from about $1.5 \%$ in the $20 \mathrm{GHz}$ region to about $2.5 \%$ in the $30 \mathrm{GHz}$ region.

The top-intensities of the absorption lines were measured as a function of temperature, going from high to low temperature and vice versa. The results of these measurements did agree with each other within experimental errors discussed above. Thus there is no difference between the measured temperature of the cell and the actual temperature of the absorbing gas. The uncertainty in the vibrational energies due to an error in the determination of $T$, can thus be neglected, provided that the temperature of the absorption cell is kept constant within $1 \mathrm{~K}$ during the time required for an intensity measurement.

At each temperature of the absorption cell the microwave power level was chosen in such a way that saturation effects did not influence the intensity ratio.

From the discussion given above it can be concluded that the relative error of the intensity ratio $R$ is caused by the error involved in the determination of the modulation depth and by the uncertainty in the value of $l_{\text {eff } 0} / l_{\text {eff }} v$. Combination of these errors results in a total error of $R(\delta R / R)$ of about $2 \%$ and $3 \%$ in the $20 \mathrm{GHz}$ and $30 \mathrm{GHz}$ regions, respectively. When vibrational energies are determined from $R$ as a function of temperature the resulting error of $E_{v}-$ $E_{0}$ depends on the number and the distribution of the measuring points, on the temperature range used and on $\delta R / R$. For the typical case of twenty measuring points uniformly distributed over the temperature range $220-320 \mathrm{~K}$, the error of $E_{r}-E_{0}^{r}$ amounts to about $300 \delta R / R \mathrm{~cm}^{-1}$. This predicted error of $E_{v}-E_{0}$ is thus in fair agreement with the experimentally found values of about $6 \mathrm{~cm}^{-1}$ (see Table I).

\section{RESULTS OF THE DETERMINATION OF THE BARRIER TO INTERNAL ROTATION FROM RELATIVE INTENSITY MEASUREMENTS}

\section{Measurements on the Molecule Methyl Silane}

The microwave spectrum of the molecule methyl silane $\left(\mathrm{CH}_{3} \mathrm{SiH}_{3}\right.$, symmetric frame-symmetric internal rotor) has been investigated by several workers $(7-9)$. Only the rotational transition $J=0 \rightarrow 1$ is found in the frequency range of our spectrometer. This rotational transition can be observed in ground as well as excited torsional states (satellite lines), and each line is split into an $A-E$ doublet. The $A-E$ doublet in the ground torsional state could not be resolved since the frequency difference between these two components is too small 
$(<50 \mathrm{kHz})$. Table II gives the frequencies of the $J=0 \rightarrow 1$ transition of $\mathrm{CH}_{3} \mathrm{SiH}_{3}$ used to determine the barrier to internal rotation from relative intensity measurements.

The energy differences between ground and excited torsional states were determined from intensity-ratio measurements as a function of temperature in the range from $225-320 \mathrm{~K}$. Adequate Stark voltage was applied to remove all the Stark components from the lines being measured. The $A-E$ doublet in the first excited torsional state was not completely resolved so that a correction for the pressure-dependent line shape was applied in order to find the intensity ratios of the individual lines with respect to the main line $(v=0)$. The results for $E_{v}-E_{0}(v=1,2,3)$, calculated from a least-square fit of the straight line relating $\ln R$ ( $R=$ Intensity ratio) and $1 / k T$ are collected in Table II.

For molecules with a symmetric internal rotor with an axis of threefold symmetry- $\mathrm{a} \mathrm{CH}_{3}$-group, for instance- the potential energy of the internal rotation $V(\alpha)$, as a function of the internal rotation angle $\alpha$, can be expanded in a Fourier series as

$$
V(\alpha)=\sum_{n}\left(V_{3 n} / 2\right)(1-\cos 3 n \alpha) .
$$

Generally to a good approximation only the first term in the summation is important. The energy difference between two torsional levels is then given by

$$
E_{v}-E_{0}=9 F \Delta b_{v} / 4
$$

in which $\Delta b_{v}$ is the difference between the corresponding eigenvalues of the Mathieu equation which is satisfied by the torsional motion and $F$ is a molecular structure parameter $(10) . \Delta b_{v}$ is related to the parameter $s$ of the Mathieu equation (11) from which the barrier height $V_{3}$ is calculated as

$$
V_{3}=9 F s / 4 \text {. }
$$

It is possible to evaluate the contribution of the first term $\left(V_{3}\right)$ as well as the

TABLE II

Frequencies of $J=0 \rightarrow 1$ Transitions of Methyl Silane and Torsional Energies

\begin{tabular}{|c|c|c|c|}
\hline \multicolumn{2}{|c|}{ Torsional state } & \multirow{2}{*}{ Frequency $(\mathrm{MHz})$} & \multirow{2}{*}{$\begin{array}{l}\text { Torsional energy } \\
E_{v}-E_{0}\left(\mathrm{~cm}^{-1}\right)\end{array}$} \\
\hline v & $A, E$ & & \\
\hline 0 & $A, E$ & 21937.92 & 0 \\
\hline 1 & $A$ & 21873.08 & $185 \pm 6$ \\
\hline 1 & $E$ & 21872.38 & $186 \pm 8$ \\
\hline 2 & $A$ & 21808.71 & $344 \pm 12$ \\
\hline 3 & $A$ & 21768.83 & $498 \pm 17$ \\
\hline
\end{tabular}
Determined from Relative Intenstty Measurements as a Function of Temperature 
second term $\left(V_{6}\right)$ to the potential energy $V(\alpha)$, from the dependence of the subsequent torsional energy levels on $v$. Herschbach (12) has described the effect of the $V_{6}$ term in the potential by adding a correction term to $\Delta b_{v}{ }^{(0)}$; thus,

$$
\Delta b_{v}=\Delta b_{v}{ }^{(0)}+\left(V_{6} / V_{3}\right) \Delta b_{v}{ }^{(1)}
$$

where the $\Delta b_{v}{ }^{(1)}$ values are also tabulated as a function of $s . V_{6} / V_{3}$ as well as $s$ can then be separately found from the point of intersection of the curves of $\left(r_{v}(s)\right.$ for different $v$-values (see Fig. 3), where

$$
G_{v}(s) \equiv\left(\Delta b_{v}-\Delta b_{v}{ }^{(0)}\right) / \Delta b_{v}{ }^{(1)} \text {. }
$$

These curves were calculated from the values of $\Delta b_{v}$ determined from the measured $E_{v}-E_{0}$ and the tables of Mathieu functions giving $\Delta b_{v}{ }^{(0)}$ and $\Delta b_{v}{ }^{(1)}$ as a function of $s(11)$. The curves do not intersect all in one point as predicted by the theory, due to the experimental errors involved in the determination of $E_{v}-E_{0}$ and thus of $\Delta b_{v}$. The mean values of $s$ and $V_{6} / V_{3}$ determined from the points of intersection (see Fig. 3) are

$$
\begin{aligned}
s & =31.0 \pm 1.2, \\
\left|V_{6} / V_{3}\right| & <0.03 .
\end{aligned}
$$

From this value of $s$ the barrier height $V_{3}$ is then finally calculated from Eq. (7).

The results obtained for $V_{3}$ and $V_{6}$ are

$$
\begin{aligned}
V_{3} & =580 \pm 22 \mathrm{~cm}^{-1}, \\
\left|V_{6}\right| & <20 \mathrm{~cm}^{-1} .
\end{aligned}
$$

Only an upper limit can be set to the value of $\left|V_{6}\right|$ due to the experimental errors in the values of $E_{v}-E_{0}$.

Our value of $V_{3}$ is in good agreement with the $558 \pm 18 \mathrm{~cm}^{-1}$ value obtained from "nonrigidity calculations" (8) and with the $585 \pm 18 \mathrm{~cm}^{-1}$ value obtained from the frequency splitting method applied to a number of asymmetric isotopic species of $\mathrm{CH}_{3} \mathrm{SiH}_{3}(9)$. It is further seen that in the case of methyl silane the RIM, as applied by us, and the FSM provide about the same accuracy in the determination of the height of the barrier to internal rotation.

\section{Measurements on the Molecule Methoxyethyne}

The microwave rotational spectrum of the molecule methoxyethyne $\left(\mathrm{HCCOCH}_{3}\right.$, asymmetric frame-symmetric internal rotor) has been investigated previously at our laboratory by D. den Engelsen (13). Besides the observation and assignment of the rotational spectrum in the ground torsional state, several rotational transitions have been assigned to the first excited torsional state. Rotational transitions in the second excited torsional state were too weak to be detected in the dense spectrum. The rotational transitions in the ground as well as in the first excited torsional state show an $A-E$ doublet 


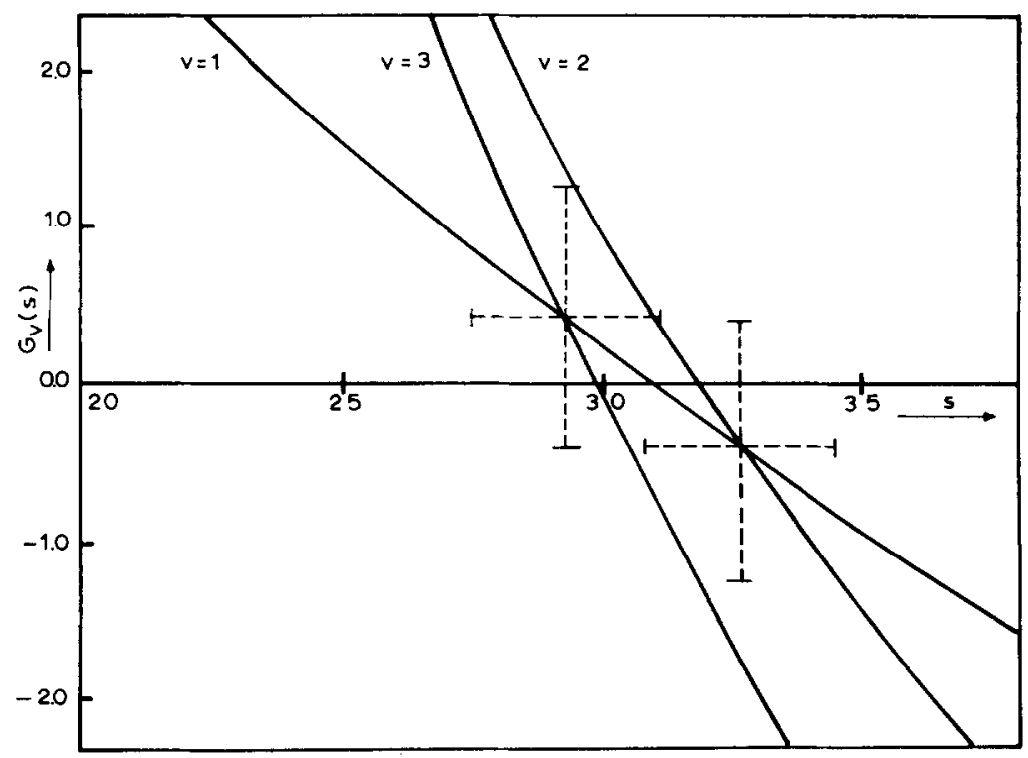

FIG. 3. Determination of the barrier parameters $V_{3}$ and $V_{6}$ from the intersecting of the curves $G_{v}(s)$ [see Eq. (10)] for $v=1,2,3$. The curve for $v=1$ is the mean curve for $v=$ $1, A$ and $v=1, E$. The errors in the points of intersection are given by the dashed lines.

structure. The height of the barrier to internal rotation has been determined from the observed $A-E$ doublet splittings (FSM). The value obtained for $V_{3}$ is

$$
V_{3}=500 \pm 10 \mathrm{~cm}^{-1} \text {. }
$$

Table III gives the frequencies of the rotational transitions used by us for the determination of the barrier to internal rotation from relative intensity measurements as a function of temperature. The measurements were performed in the range from $220-320 \mathrm{~K}$. The results of the torsional energy differences are also given in Table III.

The barrier height $V_{3}$ is calculated from the measured energy difference with the aid of Eqs (6) and (7), where it is assumed that $V_{6}=0$.

Table IV gives the results of the various barrier parameters of methoxyethyne, as follows from our work. Our value of $V_{3}$ is in good agreement with the result from the splitting measurements. The uncertainty obtained with the lattcr method arises for a great deal from the uncertainty in the value of the structure parameter $F$ (about $3 \%$ ). The error in $V_{3}$ from our measurements stems from the experimental error in the measured torsional energy and from the uncertainty in $F$. When this latter common error is left out of consideration, the error of $V_{3}$ obtained with the FSM and RIM reduces to about $1 \mathrm{~cm}^{-1}$ and $16 \mathrm{~cm}^{-1}$, 
TABLE III

Rotational Transitions (Frequencies in MHz) and Torsional Energifs (IN $\mathrm{em}^{-1}$ ) OF Methoxy fThyNe

\begin{tabular}{|c|c|c|c|c|}
\hline Transition & \multicolumn{2}{|c|}{ Ground state } & First excited state & Torsional energy \\
\hline$J_{K_{-1} K_{1}} \rightarrow J^{\prime} K_{-1}^{\prime} K_{1}^{\prime}$ & $\nu_{A}$ & $\nu_{A}-\nu_{E}$ & $\nu_{A}$ & $E_{1}-E_{0}$ \\
\hline $\begin{array}{l}3_{13} \rightarrow 4_{14} \\
4_{04} \rightarrow 4_{13}\end{array}$ & $\begin{array}{rr}38 & 173.36 \\
35 & 523.50 \\
& \text { Mea } \\
\end{array}$ & $\begin{array}{r}<0.10 \\
+17.60 \\
\end{array}$ & $\begin{array}{ll}38 & 103.23 \\
34 & 883.66\end{array}$ & $\begin{array}{l}154 \pm 5 \\
162 \pm 8 \\
156 \pm 4\end{array}$ \\
\hline
\end{tabular}

TABLE IV

Barrier Parameters of Methoxyethyne

$\begin{array}{lc}F & 6.23 \pm 0.20 \mathrm{~cm}^{-1} \\ E_{1}-E_{0} & 156 \quad \pm 4 \mathrm{~cm}^{-1} \\ \Delta b_{1} & 11.1 \pm 0.4 \\ s & 36.7 \pm 1.9 \\ V_{3} & 514 \quad \pm 20 \mathrm{~cm}^{-1}\end{array}$

respectively. Within these smaller errors the RIM and FSM also yield consistent values for the height of the barrier to internal rotation.

For the class of molecules with an asymmetric frame and a symmetric internal rotor -as $\mathrm{HCCOCH}_{3}$-in most cases the agreement between the theory and the observed spectra, including the fine structure, is particularly outstanding. Fispecially in those cases where the uncertainty in the value of $F$ is small compared with the experimental error involved in the determination of the

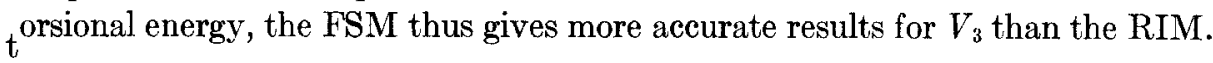

\section{Measurements on the Molecule Mono-D-methylthioethyne}

The microwave rotational spectrum of methylthioethyne $\left(\mathrm{HCCSCH}_{3}\right)$ has been investigated previously at our laboratory by D. den Engelsen (14). We have used the structure parameters of this molecule as a basis for the analysis of the rotational spectrum of the D-substituted isotopic species $\mathrm{HCCSCH}_{2} \mathrm{D}$. An accurate general theory concerning the determination of potential barriers of molecules with an asymmetric frame and an asymmetric internal rotor from the frequency splitting method, is not available yet. Information about the potential barrier to internal rotation of $\mathrm{HCCSCH}_{2} \mathrm{D}$ thus has to be obtained from the RIM.

In $\mathrm{HCCSCH}_{2} \mathrm{D}$ there are two isometric configurations, the deuterium atom may occupy a position in or outside the molecular symmetry plane (the HCCSC plane). These two rotational isomers are called the symmetrical and asymmetrical configurations, respectively. Each torsional state $v$ has three nondegenerate sublevels labeled as $v_{0}, v_{+}$and $v_{-}$. The $v_{0}$ state represents the symmetrical configuration; the $v_{ \pm}$states are the two possible asymmetrical con- 
figurations. The primary feature of the spectrum of $\mathrm{HCCSCH}_{2} \mathrm{D}$ should lie in the occurrence of three absorption lines for each rotational transition rather than two, as is the situation for molecules with a symmetric internal rotor ( $A-E$ splitting). However, in the ground torsional state $(v=0)$ the absorption lines associated with the $v_{+}$and $v_{-}$torsional substates could not be resolved, so that only a doublet was observed for each rotational transition.

Besides the observation and assignment of the rotational spectrum in the ground torsional state, several transitions of the first excited torsional state (15) were identified. In the first excited torsional state a splitting of the lines associated with the $v_{ \pm}$substates could easily be observed, so that three lines occur for each rotational transition. Table $\mathrm{V}$ shows the frequencies of the rotational transitions used for the determination of the barrier to internal rotation from relative intensity measurements.

Intensity measurements as a function of temperature in the range from 320 to $235 \mathrm{~K}$ were performed on these transitions. The energy difference between the ground and first excitcd torsional state was determined from the intensity ratios of the $5_{05} \rightarrow 5_{14}, v=0_{0}$ and $v=1_{0}$ transition, and of the $7_{07} \rightarrow 7_{16}, v=0_{ \pm}$and $v=1_{ \pm}$transitions, respectively. The results of the determination of the torsional energy difference $E_{v}-E_{0}$ are also given in Table V.

In principle, the potential barrier to internal rotation for molecules with an asymmetric internal rotor should be calculated from the torsional equation (16)

$$
\left(\mu_{T}^{(0)}{p_{\alpha}}^{2}+\mu_{T}^{(1)}(\alpha){p_{\alpha}}^{2}+V(\alpha)\right) U(\alpha)=E U(\alpha),
$$

where $\mu_{T}^{(0)}$ and $\mu_{T}^{(1)}(\alpha)$ are molecular structure parameters; $\mu_{T}^{(0)}$ contains all the $\alpha$-independent coefficients of $p_{\alpha}{ }^{2}$ and $\mu_{T}^{(1)}(\alpha)$ the $\alpha$-dependent coefficients. However, in the case of $\mathrm{HCCSCH}_{2} \mathrm{D}$ the coefficient $\mu_{T}^{(1)}(\alpha)$ amounts maximally to $2 \%$ of $\mu_{T}^{(0)}$, so that a systematic error of only a few percent is made when the potential barrier is calculated from the equation

$$
\left(\mu_{T}^{(0)} p_{\alpha}^{2}+V(\alpha)\right) U(\alpha)=E U(\alpha) .
$$

This equation is identical to the torsional equation in the case of a symmetric internal rotor. The barrier height $V_{3}$ can thus be caleulated with the aid of the usual formulas [Eqs. (6) and (7)]. The barrier parameters used for the calculation of $V_{3}$ are collected in Table VI. The uncertainty in the coefficient $\mu_{T}^{(0)}$ includes the systematic error due to the omission of $\mu_{T}^{(1)}(\alpha)$.

It is a priori expected that the barrier to internal rotation of $\mathrm{HCCSCH}_{2} \mathrm{D}$ is approximately the same as in the case of the normal methylthioethyne, since the asymmetry in the internal rotor is introduced by isotopic substitution only. Den Engelsen has determined the potential barrier of $\mathrm{HCCSCH}_{3}$ by applying the frequency splitting method (14). The result is $V_{3}=610 \pm 20 \mathrm{~cm}^{-1}$, which is in fair agreement with our value of $V_{3}$ determined from relative intensity measurements as a function of temperature. 
TABLE $V$

Rotational Transitions (Frequhncies in $\mathrm{MHz}$ ) and Torsional Energies (in $\mathrm{cm}^{-1}$ ) of Mono-D-Methylthioethyne

\begin{tabular}{|c|c|c|c|c|c|c|}
\hline \multirow{2}{*}{$\frac{\text { Transition }}{J_{K_{-1} K_{1}} \overrightarrow{J_{K_{-1}^{\prime} K_{1}^{\prime}}^{\prime}}}$} & \multicolumn{2}{|c|}{ Ground state } & \multicolumn{3}{|c|}{ First excited state } & \multirow{2}{*}{$\begin{array}{c}\begin{array}{c}\text { Torsional } \\
\text { energy }\end{array} \\
E_{1}-E_{0}\end{array}$} \\
\hline & Sym. (0) & Asym. (士) & Sym. $(0)$ & Asym & $( \pm)$ & \\
\hline $\begin{array}{l}\mathbf{5}_{0 i} \rightarrow 5_{14} \\
7_{07} \rightarrow 7_{16}\end{array}$ & $\begin{array}{ll}18 & 190.15 \\
24 & 391.07\end{array}$ & $\begin{array}{ll}17 & 651.62 \\
24 & 724.92 \\
& \text { Mean v }\end{array}$ & $\begin{array}{l}17986.87 \\
\text { lue }\end{array}$ & $24 \overline{628.50}$ & $24 \quad-534.22$ & $\begin{array}{l}153 \pm 7 \\
143 \pm 5 \\
147 \pm 4\end{array}$ \\
\hline
\end{tabular}

TABLE VI

Barrier Parameters of thl Molecule $\mathrm{HCCSCH}_{2} \mathrm{D}$

\begin{tabular}{lc} 
BARRIER PARAMETLRS OF THE MOLECULE & HCCSCH \\
\hline$h^{2} \mu_{T}(0)$ & $4.3 \pm 0.1 \mathrm{~cm}^{-1}$ \\
$E_{1}-E_{0}$ & $147 \pm 4 \mathrm{~cm}^{-1}$ \\
$\Delta b_{1}$ & $15.1 \pm 0.5$ \\
$s$ & $65 \pm 4$ \\
$V_{3}$ & $630 \pm 28 \mathrm{~cm}^{-1}$ \\
\hline
\end{tabular}

The results of our measurements on $\mathrm{HCCSCH}_{2} \mathrm{D}$ where the frequency splitting method fails, thus show that the RIM is readily applicable for determining the height of the potential barriers for molecules with an asymmetric frame and an asymmetric internal rotor.

\section{DISCUSSION AND CONCLUSIONS}

In this work we have investigated the possibilities of the method of relative intensity measurements (RIM) for the determination of the height of the potential barrier to internal rotation. The main source of error in the RIM usually is the experimental error involved in relative intensity measurements, due to the influence of frequency-dependent reflections within the absorption cell. However, as described in this work, this difficulty can be overcome for the greater part by performing intensity-ratio measurements as a function of temperature. In addition, in this way a number of unknown experimental or theoretical factors is eliminated in the determination of the potential barrier. The uncertainty in the barrier height due to experimental errors is then reduced to approximately $3 \%$. The latter error, combined with the uncertainty in the value of the moment of inertia $I_{\alpha}$ of the internal rotor $(1-3 \%)$, results in a total error in the barrier height of $3-5 \%$. This error is of the same order of magnitude as that usually obtained by the frequency splitting method (FSM) $(2-4 \%)$.

From the results given in Section $V$ it appears that in those cases where both methods can be applied, the RIM and the FSM give consistent values for the barrier height. 
In this work we applied the RIM by measuring the ratios of top intensities of absorption lines of a given rotational transition in ground as well as excited torsional states. However, when the density of the microwave spectrum of the molecule is very high, it may not always be possible to find a rotational transition that gives rise to isolated absorption lines in the ground as well as excited torsional states. It may then be possible, however, to determine the energy difference between ground and excited torsional state from the temperature dependence of the intensity ratio of a pair of suitable situated lines that belong to different rotational transitions in the ground and excited torsional state, respectively. Similarly as in the case of two absorption lines, belonging to the same rotational transition, the proportionality factor involved in the latter intensity ratio is independent of the temperature, and thus drops out when the variation of this ratio with temperature is used in the evaluation of the potential barrier.

A further improvement of the RIM may be realized by a further reduction of the reflections in the absorption cell and by an extension of the temperature range. However, the uncertainty in the potential barrier can be improved but slightly, as the uncertainty in the value of the moment of inertia of the internal rotor becomes ultimately the main limitation in the evaluation of the potential barrier.

Recently it has been shown (17) that in those cases where the torsional vibration is infrared active, a further improvement can be obtained in the accuracy of the barrier to internal rotation, from a combined infrared and microwave (FSM) determination of torsional parameters.

\section{ACKNOWLEDGMENTS}

The author would like to thank Professor Dr. C. Th. J. Alkemade for his suggestion of the experimental method in this investigation. The many helpful discussions with Dr. $\mathbf{H}$. A. Dijkerman are also deeply appreciated.

This work is part of the research program of the Stichting voor Fundamenteel Onderzoek der Materie (F.O.M.), and was made possible by financial support from the Nederlandse Organisatie voor Zuiver Wetenschappelijk Onderzoek (Z. W. O.).

ReCEIVED: March 8, 1971

\section{REFERENCES}

1. A. S. Esbitt and E. B. Wilson Jr., Rev. Sci. Instr. 34, 901 (1963); H. T. Minden and B. P. Dailey, Phys. Rev. 82, 338A (1951).

2. H. W. Harrington, J. Chem. Phys. 46, 3698 (1967); 493023 (1968).

3. A. Dymanus, Physica 25, 859 (1959).

4. G. Ruitenberg, Spectrosc. Lett. 1, 259 (1968).

b. C. II. Townes and A. L. Schawlow, "Microwave Spectroscopy," p. 445. McGraw-Hill, London, 1955; G. Ruitenberg, Thesis, State University, Utrecht, 1970.

6. S. I. Chan, T. R. Borgers, J. W. Russel, H. L. Strauss, and W. D. Gwinn, J. Chem. Phys. 44, 1103 (1966). 
7. D. R. Lide and D. K. Coles, Phys. Rev. 80, 911 (1950).

8. D. Kivelson, J. Chem. Phys. 22, 1733 (1954).

9. R. W. Kinb and L. J. Pieiree, J. Chem. Phys. 27, 108 (1957).

10. C. C. Lin and J. D. Swalen, Rev. Mod. Phys. 31, 841 (1959).

11. Tables relating to Mathieu Functions, Columbia University Press, New York, 1951.

12. D. R. Herschbach, "Tables for the Internal Rotation Problem." Department of Chemistry, Harvard University, Cambridge, MA, 1957.

18. D. den Engelsen, H. A. Dijkerman, and J. Kerssen, Rec. Trav. Chim. 84, 1357 (1965);

D. Den EnGelsen, J. Mol. Spectrosc. 30, 466 (1969).

14. D. Den Engelsen, J. Mol. Spectrosc. 30, 474 (1969); 22, 426 (1967).

15. G. Ruitenberg, to be published.

16. C. R. Quade and C. C. Lin, J. Chem. Phys. 38, 540 (1963).

17. C. E. Souter and J. L. Wood, I. Chem. Phys. 52, 674 (1970). 